Conclusion: Anti-FHL1 autoantibodies were detected in $20.5 \%$ of IIM patients. In IBM and IMNM, the presence of anti-FHL1-autoantibodies was associated with a severe myopathy as suggested by presence of dysphagia and muscle atrophy.

REFERENCES:

[1] Albrecht I, Wick C, Hallgren A, Tjarnlund A, Nagaraju K, Andrade F, et al. Development of autoantibodies against muscle-specific FHL1 in severe inflammatory myopathies. J Clin Invest. 2015;125(12):4612-24.

Disclosure of Interests: Angeles Shunashy Galindo-Feria: None declared, Begum Horuluoglu: None declared, Jessica Day: None declared, Catia Cerqueira: None declared, Susanna Proudman: None declared, Ingrid E. Lundberg Consultant of: Consulting fees from Corbus Pharmaceuticals, Inc, Grant/research support from: Research grants from Bristol Myers Squibb and Astra Zeneca, Vidya Limaye Consultant of: Scientific adviser for Actelion and Boehringer-Ingelheim, Grant/research support from: PI for clinical trials for Bayer, Boehringer-Ingelheim, Corbus, and CSL

DOI: 10.1136/annrheumdis-2021-eular.3640

\section{POS0884 \\ THE ENHANCED LIVER FIBROSIS (ELF) SCORE AS A BIOMARKER OF SKIN FIBROSIS IN SYSTEMIC SCLEROSIS}

C. Chen ${ }^{1}$, S. Yang ${ }^{1}$, Z. Jiang ${ }^{1}$, W. Wann ${ }^{1}$, H. Zou' ${ }^{1}$ M. Liang ${ }^{1} .{ }^{1}$ Huashan Hospital Affiliated to Fudan University, Department of Rheumatology, Shanghai, China

Background: Serum fibrotic markers for systemic sclerosis (SSc) remain limited. The Enhanced Liver Fibrosis (ELF) score, originally derived and validated in patients with chronic liver disease, is an algorithm combining 3 serum markers, known as procollagen type III amino terminal propeptide (PIIINP), tissue inhibitor of metalloproteinases 1 (TIMP-1), and hyaluronic acid (HA). The combined score was proved to be superior to the single components in reflecting the severity of liver fibrosis. However, the performance of ELF score and its components has not been fully validated in SSc.

Objectives: To investigate PIIINP, TIMP-1, HA, and the combined algorithm ELF score as fibrotic markers for SSc skin involvement.

Methods: Eighty SSc patients (44 dcSSc and 36 IcSSc), fulfilling the 2013 ACR/EULAR criteria with the absence of chronic liver diseases, were enrolled. Eighty age- and sex- matched healthy controls were also included. Serum PIIINP and HA levels were quantified by chemiluminescence immunoassay. Serum TIMP-1 levels were determined by enzyme-linked immunosorbent assay. The ELF score was calculated using the formula ELF score= $2.494+0.846^{*} \ln (H A)+0.735^{*} \ln (P I I I N P)+0.391^{*} \ln (T I M P-1)$. Results were correlated with clinical profiles including modified Rodnan skin score (mRSS) and interstitial lung disease (ILD).

Results: Compared with healthy controls, patients with SSc showed significantly elevated serum PIIINP (11.2 \pm 4.8 vs. $5.73 \pm 1.4 \mu \mathrm{g} / \mathrm{L}, \mathrm{p}<0.001)$, TIMP-I $(123.7 \pm 78.6$ vs. $67.8 \pm 26.5 \mathrm{ng} / \mathrm{ml}, \mathrm{p}<0.001)$, and ELF score $(10.5 \pm 0.9$ vs. $9.7 \pm 0.4, P<0.001)$. Even higher levels of PIIINP, TIMP-1, and ELF score were observed in dcSSc patients, compared with IcSSc patients $(p<0.001, p=0.024$, $p=0.003$, respectively). No significant difference was found in the levels of serum HA between patients and controls. Strong correlations were observed between mRSS and ELF score $(r=0.54, p<0.001)$, and between $m R S S$ and PIIINP $(r=0.62, p<0.001)$, whereas only weak correlations could be observed between mRSS and TIMP-1 $(r=0.28, p=0.02)$, and between mRSS and HA $(r=0.26, p=0.03)$. When stratified by ELF score, using cutoffs proposed for liver fibrosis and cirrhosis, SSc patients with ELF $<9.8$ showed the lowest mRSS on average, while patients with ELF>11.3 showed the highest $(p<0.001)$. When stratified by serum PIIINP levels, using the 25th and 75th percentiles, SSc patients with serum PIIIINP levels $<7.8 \mu \mathrm{g} / \mathrm{L}$ showed the lowest mRSS on average, while patients with PIIINP $>14.0 \mu \mathrm{g} / \mathrm{L}$ showed the highest $(\mathrm{p}<0.001)$. Neither the ELF score nor its components showed significant difference between patients with and without ILD.

Conclusion: The ELF score could be used for reflecting the severity of overall skin involvement in SSc, and serum PIIINP also increased in parallel with the increase of mRSS. Longitudinal prospective studies exploring ELF score or serum PIIINP as fibrotic markers and outcome measures of SSc are warranted.

\section{REFERENCES}

[1] Lichtinghagen R, Pietsch D, Bantel H, et al. The Enhanced Liver Fibrosis (ELF) score: Normal values, influence factors and proposed cut-off values. Journal of Hepatology. 2013; 59: 236-42.

[2] Abignano G, Blagojevic J, Bissell LA, et al. European multicentre study validates enhanced liver fibrosis test as biomarker of fibrosis in systemic sclerosis. Rheumatology. 2019; 58: 254-59.
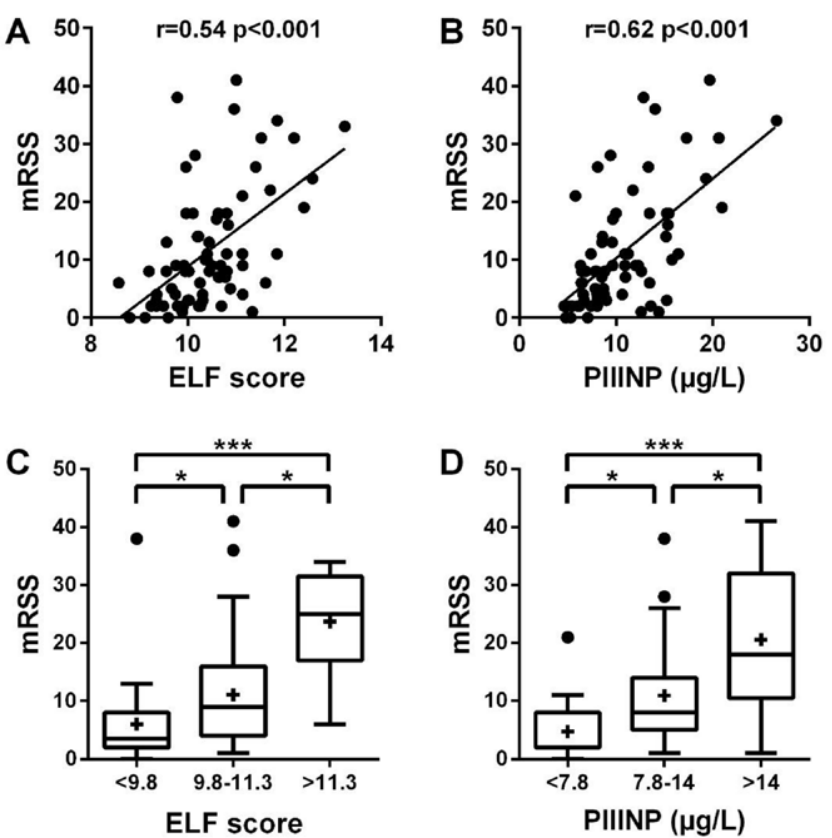

Box and whisker plots: lines from bottom to top indicated the minimum, $25^{\text {th }}$ percentile, median, $75^{\text {th }}$ percentile, and the maximum, outliers were identified by Turkey's method. Statistical analysis: (AB) Pearson's correlation and $(C D)$ Kruskal-Wallis test with Dunn's correction for multiple comparisons. + mean • outlier ${ }^{*} p<0.05{ }^{* *} p<0.01{ }^{* * *} p<0.001$

Figure 1. Correlations of mRSS with ELF score $(A)$ and serum PIIINP $(B)$ and distribution of mRSS among different ELF $(C)$ and PIIINP $(D)$ ranges.

Acknowledgements: The authors have no acknowledgements to declare Disclosure of Interests: None declared

DOI: 10.1136/annrheumdis-2021-eular.3648

\begin{tabular}{l|l}
\hline POS0885 & HIGH INCIDENCE AND MORTALITY OF \\
& PNEUMOCYSTIS JIROVECI INFECTION IN ANTI- \\
& MDA5-ANTIBODY POSITIVE DERMATOMYOSITIS: \\
& EXPERIENCE FROM A SINGLE CENTER
\end{tabular}

Q. Yan ${ }^{1}$, S. Chen ${ }^{1}$, L. Huang ${ }^{1}$, Q. Fu ${ }^{1}$, Y. Ye ${ }^{1} .{ }^{1}$ Renji Hospital, Shanghai Jiaotong University, School of Medicine, Department of Rheumatology, Shanghai, China

Background: Idiopathic inflammatory myopathies (IIM) was associated with a significantly higher risk of opportunistic infections that including Pneumocystis jiroveci pneumonia(PJP) which is potentially fatal opportunistic infection. However, no prior studies have evaluated the PJP infection in subtypes of IIM.

Objectives: To investigate the incidence rate and mortality rate of PJP infection in subgroups of IIM patients according to myopathy specific antibodies.

Methods: In the first part, we reviewed 463 consecutive patients with IIM retrospectively to analyze incidence of PJP infection. In the next part, we enrolled 30 consecutive PJP infection patients with any rheumatic disease was to identify the mortality rate and risk factors. Kaplan-Meier curve with log rank test was used to access differences in survival. Univariate and multivariate analyses were performed to identify prognostic factors using Cox regression.

Results: We found that 12(7.5\%) PJP cases occurred in 160 anti-MDA5-ab-positive DM patients, while only two $(0.7 \%)$ PJP cases were found in 303 anti-MDA5ab-negtive $D M / P M$ patients $(P<0.05)$. PJP infection typically happened in the first two months of the treatment for anti-MDA5-ab-positive DM patients who have a significant decrease in the CD4+ T cell counts and Lymphocyte counts ( $P$ $<0.05)$. Only two (16.7\%) anti-MDA5-ab-positive DM patients recover from PJP with lethally higher mortality than those PJP infection with other rheumatic diseases $(83.3 \%$ vs. $38.9 \%, P<0.05)$. We found no association between the time to anti-PJP treatment and treatment outcomes in anti-MDA5-ab-positive DM; ye we confirmed in PJP infection with other rheumatic diseases that anti-PJP treatment within 6 days crucially increased the survival $(P<0.05)$

Conclusion: PJP infection has alarming high incidence and mortality in antiMDA5-ab-positive DM patients. Unlike PJP infection with other rheumatic diseases, timely treatment for PJP doesn't improve the prognosis of this particular subtype. Therefore, the necessity of further study of PJP prophylaxis treatment in anti-MDA5-ab-positive DM patients is verified. 


\section{REFERENCES}

[1] Hsu CY, et al. Comparing the burdens of opportunistic infections among patients with systemic rheumatic diseases: a nationally representative cohort study. ARTHRITIS RES THER 2019, 21(1):211.

Acknowledgements: The authors thank Dr. An Sun,

Disclosure of Interests: None declared

DOI: 10.1136/annrheumdis-2021-eular.3693

\section{POS0886 COULD BE INTERSTITIAL MYOCARDITIS A FEATURE OF THE ANTISYNTHETASE SYNDROME?}

A. Gil-Vila ${ }^{1}$, G. Burcet ${ }^{2}$, A. Anton-Vicente ${ }^{1}$, D. Gonzalez-Sans ${ }^{1}$, A. NuñezConde $^{1}$, E. Trallero-Araguás ${ }^{3}$, J. L. Reyes-Juárez² ${ }^{2}$ C. P. Simeón-Aznar ${ }^{1}$ A. Selva-O'callaghan ${ }^{1}{ }^{1}$ Vall d'Hebron University Hospital, Internal Medicine, Barcelona, Spain; ${ }^{2}$ Vall d'Hebron University Hospital, Cardiovascular Imaging, Radiology, Barcelona, Spain; ${ }^{3}$ Vall d'Hebron University Hospital, Rheumatology, Barcelona, Spain

Background: Antisynthetase syndrome (ASS) is characterized by inflammatory myopathy, interstitial lung disease, arthritis, mechanical hands and Raynaud phenomenon, among other features. Recent studies have shown that idiopathic inflammatory myopathies (IIM) may develop cardiac involvement, either ischemic (coronary artery disease) or inflammatory (myocarditis). We wonder if characteristic lung interstitial involvement (interstitial lung disease) that appears in patients with the ASS may also affect the myocardial interstitial tissue. New magnetic resonance mapping techniques could detect subclinical myocardial involvement, mainly as edema (increase extracellular volume in interstitium and extracellular matrix), even in the absence of visible late Gadolinium enhancement (LGE). Objectives: Our aim was to describe the presence of interstitial myocarditis in a group of patients with ASS.

Methods: Cross-sectional, observational study performed in a tertiary care center. We included 13 patients diagnosed with ASS (7 male, 53\%, mean (SD) age at diagnosis 56,8 years $( \pm 11,8)$ ). The patients were consecutively selected from our outpatient myositis clinic. Myositis specific and associated antibodies were performed by means of line immunoblot (EUROIMMUN ${ }^{\odot}$ ). Cardiac magnetic resonance (CMR) was performed on all patients. The study protocol includes functional cine magnetic resonance and standard late gadolinium enhancement (LGE), as well as novel parametric T1 and T2 mapping sequences (modified look locker inversion recovery sequences - MOLLI) with extracellular volume (ECV) calculation 20 minutes after the injection of a gadolinium-based contrast material. Results: CMR could not be performed in one patient due to anxiety. All patients studied (12) had a normal biventricular function, without alteration of segmental contraction. A third (4 out of $12,33 \%$ ) of the studied patients showed elevated T2 myocardial values without focal LGE, half of them (2/4) with an elevated ECV, consistent with myocardial edema. Two patients with normal T2 values showed unspecific LGE focal patterns, one in the right ventricle union points and another with mild interventricular septum enhancement (Figure 1). None of the patients studied refer any cardiac symptomatology. All the four patients with T2 mapping alterations (100\%) had interstitial lung involvement, but only 4 out of $8(50 \%)$ of the rest ASS patients without T2 mapping positivity. The autoimmune profile was as follows: 10 anti-Jo1/Ro52, 1 anti-EJ/Ro52, 2 anti-PL12.

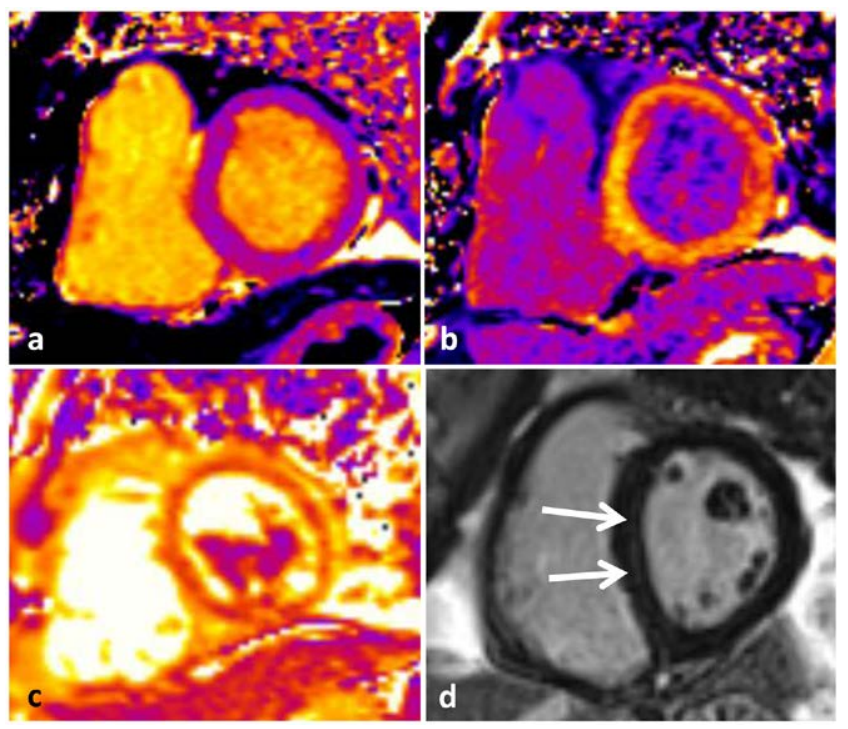

Figure 1. Cardiac magnetic resonance images from ASS patients.
Conclusion: Myocarditis, although subclinical, appears to be a feature in ASS patients. T1 and T2 mapping sequences might be valuable to detect and monitor subclinical cardiac involvement in these patients. The possibility that the same etiopathogenic mechanism may be involved in the interstitial tissue in lung and myocardium is raised. More studies must be done in order to assert the prevalence of myocarditis in ASS.

REFERENCES:

[1] Dieval C et al. Myocarditis in Patients With Antisynthetase Syndrome: Prevalence, Presentation, and Outcomes. Medicine (Baltimore). 2015 Jul;94(26):e798.

[2] Myhr KA, Pecini R. Management of Myocarditis in Myositis: Diagnosis and Treatment. Curr Rheumatol Rep. 2020 Jul 22; 22:49.

[3] Sharma K, Orbai AM, Desai D, Cingolani OH, Halushka MK, Christopher-Stine L, Mammen AL, Wu KC, Zakaria S. Brief report: antisynthetase syndrome-associated myocarditis. J Card Fail. 2014 Dec;20(12): 939-45.

Disclosure of Interests: None declared DOI: 10.1136/annrheumdis-2021-eular.3696

\section{POS0887 TRANSCRANIAL ELECTRICAL STIMULATION IS SAFE AND EFFICIENT IN PATIENTS WITH SYSTEMIC AUTOIMMUNE MYOPATHIES}

L. F. Adsuara de Sousa ${ }^{1}$, R. G. Misse ${ }^{1}$, L. De Macedo Dos Santos ${ }^{2}$, A. Fontes Baptista $^{3}$, C. Tanaka ${ }^{2}$, J. M. D'andréa Greve ${ }^{4}$, S. Katsuyuki Shinjo ${ }^{1} .{ }^{1}$ Faculdade de Medicina FMUSP, Universidade de Sao Paulo, Division of Rheumatology, Sao Paulo, Brazil; ' 2 Speech Therapy and Occupational Therapy, Hospital das Clinicas HCFMUSP, Faculdade de Medicina, Universidade de Sao Paulo, Department of Physical Therapy, Sao Paulo, Brazil; ${ }^{3}$ Universidade Federal do ABC, Center for Mathematics, Computing and Cognition, Santo Andre, Brazil; ${ }^{4}$ Institute of Orthopedics and Traumatology, Hospital das Clinicas HCFMUSP, Faculdade de Medicina, Universidade de Sao Paulo, Laboratory of Movement Studies, Sao Paulo, Brazil

Background: There is currently few information regarding rehabilitation in patients with systemic autoimmune myopathies (SAMs). Transcranial direct current stimulation (tDCS) has shown promising results for the motor performance of healthy individuals as for patients with, e.g., post-stroke hemiparetic limbs. Objectives: The present study was aimed to assessing the safety and efficiency of tDCS in patients with SAMs.

Methods: This study is a prospective, randomized, sham controlled, double blind, and clinical trial with ethical approval. Eighteen adult patients with dermatomyositis, polymyositis, antisynthetase syndrome or immune-mediated necrotizing myopathies in remission or with minimal disease activity were enrolled from 2018 to 2019. Patients were allocated randomly in two groups to receive sham or active tDCS with $2 \mathrm{~mA}$ amplitude submitted for 20 minutes for three consecutive days. The $5 \times 7 \mathrm{~cm}$ sponge-electrodes were positioned with the anode over the left $(\mathrm{C} 1)$ or right $(\mathrm{C} 2)$ - contralateral to the dominant limb, whereas the cathode over the FP2 or FP3, respectively (10-10 EEG electrodes placement). The groups were evaluated in four moments: pre-stimulation, and 30 minutes, 3 weeks and 8 weeks post-tDCS. They were evaluated in the different moments with International Myositis Assessment and Clinical Studies Group set scores, Short-Form health survey (SF-36), state-trait anxiety inventory (STAI), Beck depression inventory (BDI), timed up-and-go test (TUG), time-stands test (TST), isokinetic extension and flexion testing of bilateral knee and elbow. A specific security questionnaire for tDCS was used after the active or sham stimulation in all patients.

Results: The demographic data, kind of myositis, disease duration, and disease status (all with low disease activity) were comparable between both active and sham tDCS groups. After interventions, there was improvement of values of patient's VAS $(P=0.042)$ and serum levels of creatine phosphokinase $(P=0.005)$, independent of the group. Moreover, in active tDCS group, the physical aspects of SF-36 in week $8(P<0.001)$, mean and better TST at each evaluation $(P<0.001)$, absolute peak tork $(P<0.001)$ and peak tork adjusted for body weight values $(P<0.001)$ of stimulated inferior limb extension also improved. No differences were observed in the STAI, BDI, or TUG in both groups. The patients adherence to the protocol was $100 \%$ and no adverse event was reported, including disease relapsing

Conclusion: This unprecedented study evidences the safety of tDCS, besides the potential efficiency in improving rehabilitation of tDCS in SAMs. More studies with a large samples and period of tDCS sessions are necessaries to corroborate with the present study.

\section{REFERENCES:}

[1] Lundberg IE, et al. 2017 European league against rheumatism/ American College of Rheumatology classification criteria for adult and juvenile idiopathic inflammatory myopathies and their major subgroups. Arthritis Rheum. 2017:69:2271-82. 